\title{
The Effect of Inharmonic Partials on Pitch of Piano Tones
}

\author{
Brian E. Anderson
}

William J. Strong

Follow this and additional works at: https://scholarsarchive.byu.edu/facpub

Part of the Astrophysics and Astronomy Commons, and the Physics Commons

\section{Original Publication Citation}

B. E. Anderson and W. J. Strong, "The effect of inharmonic partials on pitch of piano tones," J. Acoust. Soc. Am., 117, 3268-3272 (25).

\section{BYU ScholarsArchive Citation}

Anderson, Brian E. and Strong, William J., "The Effect of Inharmonic Partials on Pitch of Piano Tones" (2005). Faculty Publications. 1002.

https://scholarsarchive.byu.edu/facpub/1002 accepted for inclusion in Faculty Publications by an authorized administrator of BYU ScholarsArchive. For more information, please contact ellen_amatangelo@byu.edu. 


\title{
The effect of inharmonic partials on pitch of piano tones ${ }^{\text {a) }}$
}

\author{
Brian E. Anderson ${ }^{\text {b) }}$ and William J. Strong \\ Department of Physics and Astronomy, N-283 ESC, Brigham Young University, Provo, Utah 84602
}

(Received 4 November 2004; revised 17 January 2005; accepted 8 February 2005)

\begin{abstract}
Piano tones have partials whose frequencies are sharp relative to harmonic values. A listening test was conducted to determine the effect of inharmonicity on pitch for piano tones in the lowest three octaves of a piano. Nine real tones from the lowest three octaves of a piano were analyzed to obtain frequencies, relative amplitudes, and decay rates of their partials. Synthetic inharmonic tones were produced from these results. Synthetic harmonic tones, each with a twelfth of a semitone increase in the fundamental, were also produced. A jury of 21 listeners matched the pitch of each synthetic inharmonic tone to one of the synthetic harmonic tones. The effect of the inharmonicity on pitch was determined from an average of the listeners' results. For the nine synthetic piano tones studied, pitch increase ranged from approximately two and a half semitones at low fundamental frequencies to an eighth of a semitone at higher fundamental frequencies. (C) 2005 Acoustical Society of America. [DOI: $10.1121 / 1.1882963]$
\end{abstract}

PACS numbers: 43.75.Cd, 43.66.Hg [ADP]

Pages: $3268-3272$

\section{INTRODUCTION}

Several studies have investigated various subjective behaviors of complex inharmonic pianolike tones. Fletcher et al. (1962) found, through subjective testing, that inharmonicity in piano tones contributes a sense of warmth. In 1983, Peters et al. (1983) had listeners match components in complex harmonic pianolike tones to a single sine wave to determine the effect on pitch. Moore et al. (1985a) found, through subjective testing, that a complex tone consisting of one slightly mistuned partial resulted in a linear residue pitch shift for small mistunings. Moore et al. (1985b) also used this type of subjective testing to determine detection thresholds and the importance of each partial. They suggested that inharmonicity is audible for lower piano tones. Moore et al. (1993) determined listeners' psychoacoustic abilities to hear individual partials in inharmonic complex tones. Conklin (1996) wrote a series of tutorial papers, the third of which is a summary of research investigations on the subject of inharmonicity. Rocchesso et al. (1999) studied "The influence of accurate reproduction of inharmonicity on the perceived quality of piano tones." And most recently, Galembo et al. (2001) determined the perceived effect of having various kinds of starting phases for synthetic tones.

This study investigates the effect of inharmonicity on pitch by having listeners match complex inharmonic pianolike tones (based on measured tones) to complex harmonic pianolike tones (based on ideal string theory). Nine piano tones from a standard upright piano were recorded and analyzed. The analysis determined various parameters for as many partials as could be resolved for each of the ninerecorded tones. From this analysis, a synthetic inharmonic tone and a set of harmonic tones with different fundamental

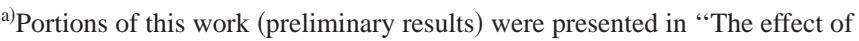
inharmonic partials on pitch of piano tones," 143rd Meeting: Acoustical Society of America, Pittsburgh, PA, June 2002.

${ }^{b}$ Present address: Graduate Program in Acoustics, The Pennsylvania State University, P.O. Box 30, State College, PA 16802. Electronic mail: bea3@email.byu.edu
}

frequencies were produced for each of the nine tones. A jury of listeners matched each synthetic inharmonic tone to one of the synthetic harmonic tones among the corresponding set. The effect of inharmonicity on pitch was determined from the average fundamental frequency of harmonic tones matched to an inharmonic tone.

\section{TONE GENERATION}

\section{A. Recording}

In order to study the effects of inharmonicity, a readily available Yamaha Upright Piano (P22 L. OAK) was used for the study. A Larson Davis 2540 pressure microphone was used with a Larson Davis 900B Preamplifier. The 900B preamplifier was then connected to a Larson Davis 2200C Microphone Power Supply and Instrumentation Amplifier. The output of the 2200C went into an Aphex Amplifier 124A. A Panasonic DAT recorder was used to record the output signal from the amplifier.

The recording was done in a large classroom that had been acoustically treated to reduce reverberation. Recordings were done at times when ambient levels could be maintained without interruptions. With this recording environment and the recording setup, it was possible to maintain an overall $\mathrm{S} / \mathrm{N}$ ratio of at least $30 \mathrm{~dB}$. The Yamaha Upright Piano has a removable cover that allowed for better exposure of the soundboard on the player's side of the piano. The microphone was placed about 6 in. from the soundboard.

Tones were recorded in the lower three octaves of the piano where the effects of inharmonicity are greater. Nine tones (A0, C1, E1, A1, C2, E2, A2, C3, and E3) were individually played and recorded. An effort was made to strike each piano key with the same amount of force. Tones were recorded until their overall sound pressure level decayed to the level of ambient noise in the room. The resulting recorded tones had durations ranging from 10 to $15 \mathrm{~s}$. Finally, the tones recorded on the DAT were digitally transferred onto a $\mathrm{CD}$. 


\section{B. Analysis}

The tones were played on a CD player whose output was directly connected to the input of a Hewlett-Packard dynamic signal analyzer and analyzed in order to obtain the desired partial frequencies. Some of the piano tones had as many as 56 resolvable partials that were obtained in the analysis. In this analysis, it was assumed that the partial frequency values remained constant over time, which, from the tones' spectrograms, proved to be a valid assumption.

The recorded tones were then imported into MATLAB for further analysis. The resolvable frequency values of the partials obtained from the dynamic signal analyzer were used in the MATLAB analysis. The analysis determined the relative initial amplitudes and decay rates for as many partials as could be resolved for each of the nine-recorded tones. An attack portion was also determined for each tone analyzed. Fourier analysis was used to determine the amplitude of each partial, over the period of the fundamental frequency, repeated for $10 \mathrm{~s}$. A linear curve fit was applied to a logarithmic representation of the amplitude versus time data for each partial, from which the initial amplitude and decay rate were obtained. The attack portion of each recorded tone was determined as the time it took from the onset of the tone to reach peak amplitude. The initial phase $\phi_{n}$ for each partial was assigned a random value between 0 and $2 \pi$. The results of the analysis for each of the nine tones consisted of frequencies of the partials, $f_{n}$, amplitudes of the partials, $A_{n}$, decay rates of the partials, $\delta_{n}$, and initial phases, $\phi_{n}$.

\section{Synthesis}

Based on the analysis, a synthetic inharmonic tone was produced for each of the nine tones analyzed. Each synthetic inharmonic tone was generated based on the following equation,

$$
y_{\text {inharmonic }}=A_{n} e^{-\delta_{n} t} \sin \left(2 \pi f_{n} t+\phi_{n}\right),
$$

where the variables are the results from the analysis.

A series of 10 to 30 harmonic tones was generated for comparison with each inharmonic tone. Partial frequencies of the harmonic tones were integer multiples of the fundamental. Each synthetic harmonic tone was generated based on the following equation,

$$
y_{\text {harmonic }}=A_{n} e^{-\delta_{n} t} \sin \left(2 \pi n f_{1} t+\phi_{n}\right),
$$

where the variables, with the exception of the fundamental frequency $f_{1}$, are results from the analysis. The fundamental frequency for each harmonic tone in the series was incremented in a ratio of 1.005 (approximately a twelfth of a semitone).

A linearly increasing (from zero to the maximum amplitude) attack portion, determined from the analysis procedure, was applied to each synthetic tone. For each of the synthetic harmonic piano tones, an audio CD track was created consisting of the corresponding synthetic inharmonic tone presented first followed by the synthetic harmonic tone. Much of the synthesis procedure was chosen based on previous work done by Anderson (2002).
TABLE I. Curve-fitted inharmonicity coefficients for each of the nine measured piano tones.

\begin{tabular}{cc}
\hline Piano note & $\begin{array}{c}\text { Inharmonicity } \\
\text { coefficient }\end{array}$ \\
\hline A0 & 0.000453 \\
C1 & 0.000319 \\
E1 & 0.000231 \\
A1 & 0.000144 \\
C2 & 0.000130 \\
E2 & 0.000108 \\
A2 & 0.000111 \\
C3 & 0.000129 \\
E3 & 0.000110 \\
\hline \hline
\end{tabular}

\section{Inharmonicity coefficient}

In 1964, Fletcher published a theoretical derivation of an equation governing the shift in partial frequencies of piano strings due to the inherent stiffness in piano strings. Fletcher (1964) gave the following equation for predicting partial frequencies once the fundamental frequency, which itself is affected by the stiffness, is known:

$$
f_{n}=n f_{1}\left[\frac{\left(1+B n^{2}\right)}{(1+B)}\right]^{1 / 2},
$$

where $B$ represents the inharmonicity coefficient. Using this equation, an estimate for the inharmonicity coefficient was determined, using the method of least squares, for each of the nine measured tones.

The least-squares curve fit for the inharmonicity coefficient included a heavier weighting on the lower partials. For the $n$th partial, the least squares error weighting $W(n)$ is computed as

$$
W(n)=N+1-n,
$$

where $N$ is the total number of partials for the given tone. The resulting weighting is a linearly decreasing function. The results for the curve fitted $B$ values may be found in Table I. Each curve fit result represented the increasing partial frequency values fairly accurately, with a slight tendency to underestimate the lower partials and overestimate the higher partials. The $B$ values were determined in order to investigate the correlation between the pitch shift due to inharmonicity and the inharmonicity coefficient for each measured piano tone.

\section{METHOD}

\section{A. Subjects}

Twenty-one subjects volunteered from among the Physics 167 "Descriptive Acoustics of Music and Speech" course offered at Brigham Young University. Students who participated in the study were given extra credit towards their grade in that course. The average age of participants was 20.7 years, with $76 \%$ female; $33 \%$ had taken a recent hearing test. Participants had an average of 6.6 years of piano playing experience, with an average of 9.1 years total musical instrument experience. Table II shows the data for the listeners, listed according to piano playing experience. 
TABLE II. Data for volunteer listeners who participated in inharmonicity perceptual study.

\begin{tabular}{cccccc}
\hline \hline $\begin{array}{c}\text { Listener } \\
\text { rank }\end{array}$ & $\begin{array}{c}\text { Age } \\
\text { (years) }\end{array}$ & Sex & $\begin{array}{c}\text { Taken } \\
\text { recent } \\
\text { hearing } \\
\text { test }\end{array}$ & $\begin{array}{c}\text { Years } \\
\text { experience } \\
\text { playing the } \\
\text { piano }\end{array}$ & $\begin{array}{c}\text { Total years } \\
\text { of musical } \\
\text { experience }\end{array}$ \\
\hline 1 & 21 & F & N & 15 & 15 \\
2 & 20 & F & N & 15 & 15 \\
3 & 20 & F & N & 14 & 14 \\
4 & 20 & F & Y & 13 & 13 \\
5 & 19 & F & N & 12 & 12 \\
6 & 20 & F & Y & 11 & 11 \\
7 & 24 & F & N & 10 & $10+$ \\
8 & 19 & F & N & 10 & 10 \\
9 & 20 & F & N & 8 & 10 \\
10 & 22 & M & N & 6 & 12 \\
11 & 19 & F & N & 6 & 8 \\
12 & 20 & F & Y & 5 & 8 \\
13 & 17 & F & Y & 5 & 8 \\
14 & 18 & F & Y & 5 & 7 \\
15 & 20 & F & Y & 2 & 5 \\
16 & 20 & F & Y & 1 & 8 \\
17 & 22 & M & N & 0 & 13 \\
18 & 24 & M & N & 0 & $10+$ \\
19 & 25 & M & N & 0.5 & 0.5 \\
20 & 25 & M & N & 0 & 3 \\
21 & 19 & F & N & 0 & 0 \\
Average & 20.7 & $76 \%$ F & $33 \%$ Y & 6.6 & 9.1 \\
\hline \hline & & & & & \\
\hline
\end{tabular}

\section{B. Stimuli}

Listeners were given an audio CD player and a set of Sony Studio Monitor/Dynamic Stereo Headphones (model MDR-7506) to playback the tone pairs. Listeners set the volume levels according to their comfort levels. Listeners were placed in a quiet, isolated, semi-anechoic chamber facility located at Brigham Young University. Listeners were free to listen to tone pair CD tracks in any order they chose and as many times as needed. Each listener was instructed to try and find a tone pair, for each of the nine sets, that they perceived as having the closest pitch match. If no match was found among a given set of tone pairs, they were instructed to indicate whether the harmonic tones needed to be higher or lower in pitch in order to find a match; these responses were not included in further analysis.

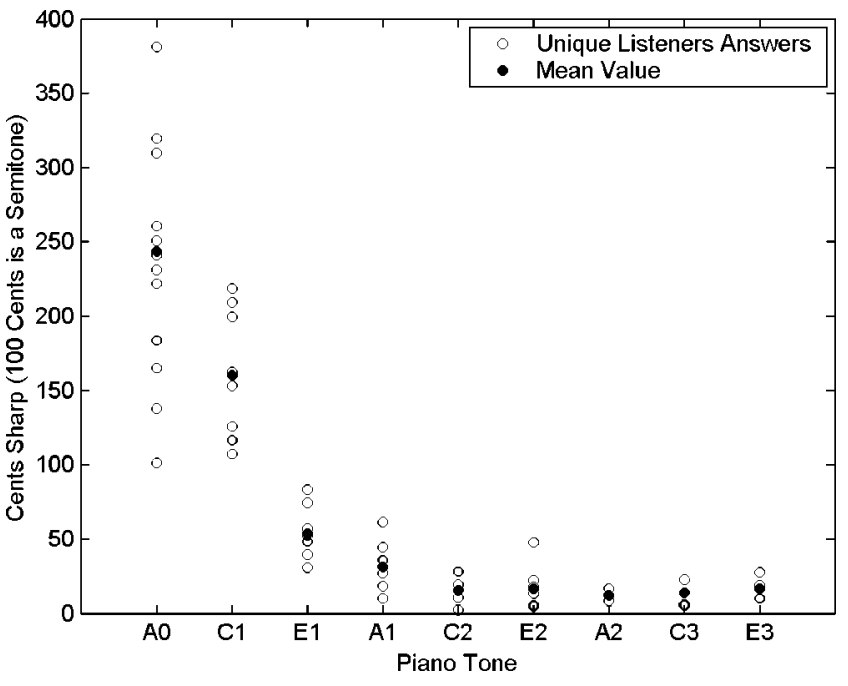

FIG. 1. Results for pitch matching study. Open circles represent unique listeners' answers and closed circles represent the average match for each piano tone set.

\section{RESULTS}

Figure 1 shows a scatter plot of individual responses and their average for each tone. (Overlapping individual responses are not apparent in the figure.) The average pitch shift due to inharmonicity was greater in lower frequency piano tones. It can also be seen that the spread or deviation is much greater for lower tones. Table III tabulates the average results of the listening tests. Table III also shows deviation values.

In order to compare the listeners' results for the perceived shift in pitch due to inharmonicity with the least squares fitted values for the inharmonicity coefficients, each set of results was normalized by their respective mean value. A plot of the two normalized sets of results may be found in Fig. 2. Also contained in Fig. 2 is a plot of the normalized relative standard deviation of listeners' results.

A plot of the average perceived cents sharp values divided by the fitted inharmonicity coefficients versus fundamental frequency results in an exponentially decreasing function. This function was least squares curve fitted to an equation of exponential form with an added offset constant.

TABLE III. Average results of listening tests. The actual fundamental and the average matched fundamental frequency values are given in Hertz. Standard deviation values are determined from the matched fundamental frequency values. The relative standard deviation values are defined as the standard deviation values divided by their respective mean values (then multiplied by 100 to represent a percentage).

\begin{tabular}{|c|c|c|c|c|c|}
\hline $\begin{array}{l}\text { Piano } \\
\text { note }\end{array}$ & $\begin{array}{c}\text { Actual } \\
\text { fundamental } \\
(\mathrm{Hz})\end{array}$ & $\begin{array}{c}\text { Average } \\
\text { perceived } \\
\text { fundamental } \\
(\mathrm{Hz})\end{array}$ & $\begin{array}{c}\text { Cents sharp } \\
((\text { Ratio }-1) / 0.059)\end{array}$ & $\begin{array}{c}\text { Standard } \\
\text { deviation } \\
(\mathrm{Hz})\end{array}$ & $\begin{array}{l}\text { Relative } \\
\text { standard } \\
\text { deviation }\end{array}$ \\
\hline A0 & 26.750 & 30.597 & 243.8 & 13.71 & 44.81 \\
\hline $\mathrm{C} 1$ & 32.125 & 35.161 & 160.2 & 3.39 & 9.63 \\
\hline E1 & 40.375 & 41.649 & 53.5 & 3.29 & 7.89 \\
\hline A1 & 54.125 & 55.131 & 31.5 & 2.53 & 4.59 \\
\hline $\mathrm{C} 2$ & 64.500 & 65.094 & 15.6 & 1.99 & 3.06 \\
\hline E2 & 81.375 & 82.162 & 16.4 & 2.21 & 2.69 \\
\hline A2 & 108.625 & 109.416 & 12.3 & 1.15 & 1.05 \\
\hline C3 & 128.500 & 129.559 & 14.0 & 1.27 & 0.98 \\
\hline E3 & 163.250 & 164.830 & 16.4 & 1.76 & 1.07 \\
\hline
\end{tabular}




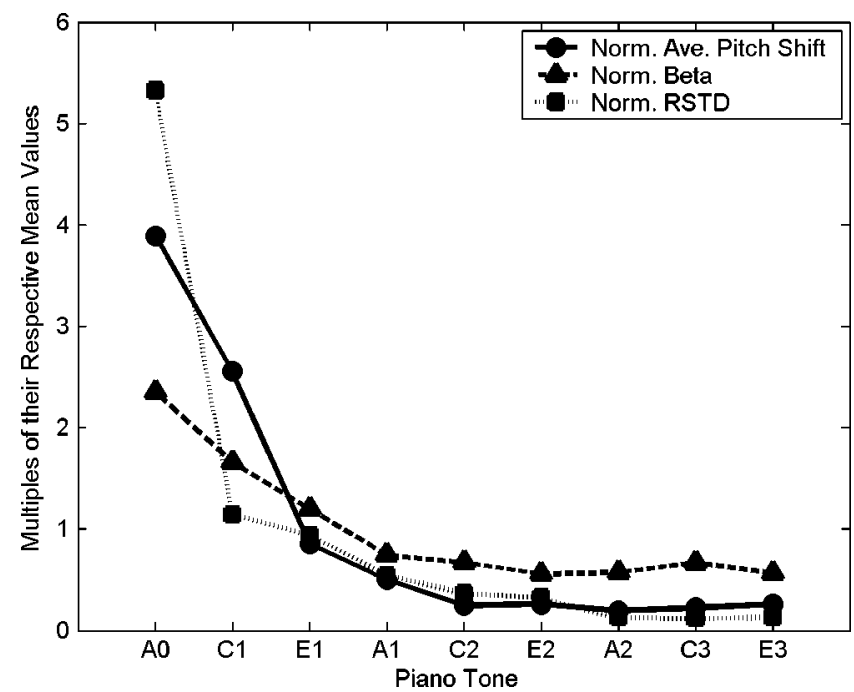

FIG. 2. Normalized average results for pitch matching study, normalized results for inharmonicity coefficient determination, and normalized relative standard deviation results. A value of 1.0 on this plot represents the average value for each data set.

The resulting coefficients for the curve fit resulted in the following equation,

$$
\begin{aligned}
\text { CentsSharp }= & B[271 \times \exp (-0.0681 \times f)+12.5] \\
& \times 10000,
\end{aligned}
$$

where CentsSharp is the perceived cents sharp value, $B$ is the inharmonicity coefficient, and $f$ is frequency in Hz. Figure 3 shows the average cents sharp values divided by the fitted inharmonicity coefficients versus fundamental frequency, along with the optimum exponential curve fit result. The "error bars" represent the standard deviation among listeners' results. It should be noted that for frequencies above around $60 \mathrm{~Hz}$, the relationship between the perceived cents sharp value and the inharmonicity coefficient becomes equivalent to a linear relationship according to the following equation,

$$
\text { CentsSharp }(f>60 \mathrm{~Hz})=125000 \times B \text {. }
$$

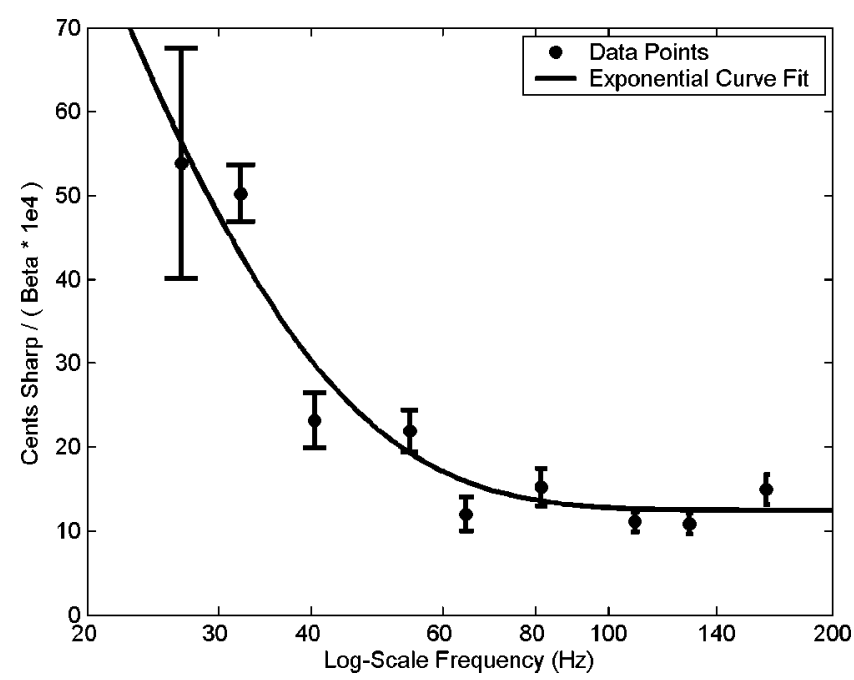

FIG. 3. Filled circles represent average perceived cents sharp values divided by the curve fitted inharmonicity coefficients (scaled by 10000 ). The solid line represents the least-squares exponential curve fit to the filled circle values. The "error bars" represent the standard deviation among listeners' results.

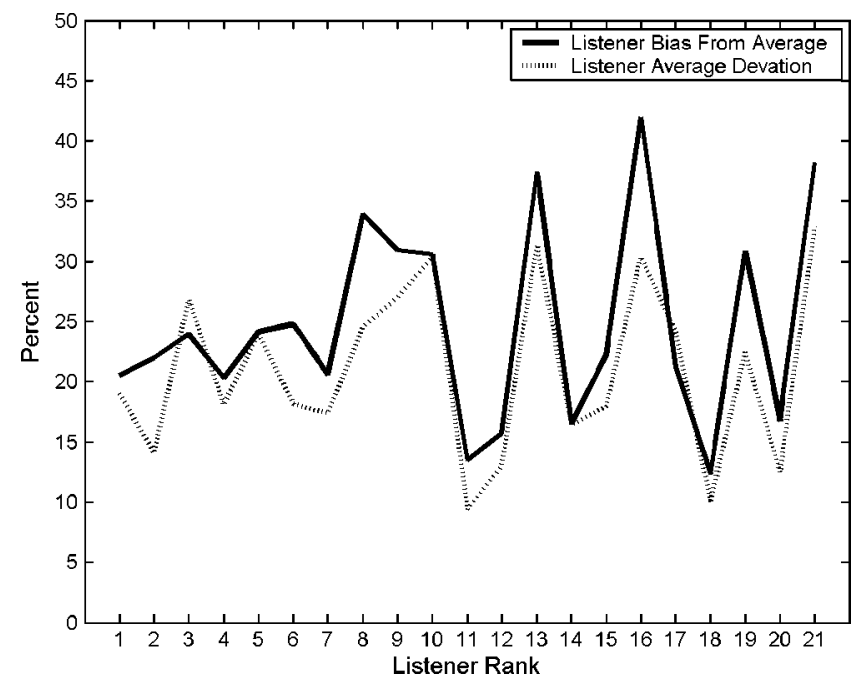

FIG. 4. Comparison across individual listeners of average listener's bias deviation from the average responses and average, across all nine piano tones, listener's relative standard deviation of bias values.

An analysis of each individual listener's results was done in order to determine the dependence on listeners' musical experience. The absolute value of the bias "error," between each listener's answer and the average value for the given tone, was determined. These values were then averaged across the nine piano tone sets for each listener to determine an average bias error value (relative to the average answers) for the given listener. The standard deviation of the bias errors was also determined and averaged across each listener to create a relative standard deviation value. A plot of the bias results and the deviation results may be found in Fig. 4.

\section{DISCUSSION}

From Fig. 2 it is apparent that the average amount of pitch shift perceived by the jury of listeners is strongly correlated with the inharmonicity coefficient values in the upper seven piano tones studied (E1, A1, C2, E2, A2, C3, and E3). For the lower two piano tones, $\mathrm{A} 0$ and $\mathrm{C} 1$, the increase in pitch shift relative to the increase in inharmonicity rises significantly.

This study also found an increase in deviation of listeners' perceived matching results for lower fundamental frequency piano tones. This might have some explanation in the well-known psychoacoustic phenomenon that pitch perception ability decreases at lower frequencies.

It was intuitively compelling to find that a given listener's ability to find a pitch match did not depend on their piano musical training. Any given listener tended to have relatively equal probability of perceiving the average of the total listener's pitch matches.

\section{CONCLUSIONS}

The perceived pitch due to inharmonicity in piano tones correlated with the inharmonicity coefficient for a given piano tone, although the correlation was less pronounced for the lowest two tones. The deviation among listeners' pitch matches increased at lower frequencies and showed correla- 
tion with pitch shift. A given listeners' musical experience of playing the piano did not correlate with the ability to perceptually match piano tones.

The correlation between the pitch shift and the inharmonicity coefficient for the higher frequency piano tones should not be a surprising result. The significant rise in the pitch shift trend relative to the inharmonicity coefficient values for the lowest two tones, however, is a surprising result. The increased pitch shift found in the lower two piano tones should be further studied and extended to the entire lowest octave on a piano (roughly A0 to $G \# 1$ ). The pitch shift found in piano tone $\mathrm{A} 0$ was determined to be nearly 250 cents, two and a half steps, or two and a half semitones.

\section{ACKNOWLEDGMENTS}

Portions of this project were supported by the Brigham Young University Acoustics Research Endowment Fund and by the Brigham Young University Physics and Astronomy Department. Thanks are also due to Michael Thompson, who helped on the project while at Brigham Young University.
Anderson, B. E. (2002). "The effect of inharmonic partials on pitch of piano tones," J. Acoust. Soc. Am. 111, 2394.

Conklin, Jr., H. A. (1996). "Design and tone in the mechanoacoustic piano. Part III. Piano strings and scale design," J. Acoust. Soc. Am. 100, 12861298.

Fletcher, H. (1964). "Normal vibration frequencies of a stiff piano string," J. Acoust. Soc. Am. 36, 203-209.

Fletcher, H., Blackham, E. D., and Stratton, R. (1962). "Quality of piano tones," J. Acoust. Soc. Am. 34, 749-761.

Galembo, A., Askenfelt, A., Cuddy, L. L., and Russo, F. A. (2001). "Effects of relative phases on pitch and timbre in the piano bass range," J. Acoust. Soc. Am. 110, 1649-1666.

Moore, B. C. J., and Ohgushi, K. (1993). "Audibility of partials in inharmonic complex tones," J. Acoust. Soc. Am. 93, 452-461.

Moore, B. C. J., Glasberg, B. R., and Peters, R. W. (1985a). "Relative dominance of individual partials in determining the pitch of complex tones," J. Acoust. Soc. Am. 77, 1853-1860.

Moore, B. C. J., Peters, R. W., and Glasberg, B. R. (1985b). "Thresholds for the detection of inharmonicity in complex tones," J. Acoust. Soc. Am. 77, 1861-1867.

Peters, R. W., Moore, B. C. J., and Glasberg, B. R. (1983). "Pitch of components of complex tones," J. Acoust. Soc. Am. 73, 924-929.

Rocchesso, D., and Scalcon, F. (1999). "Bandwidth of perceived inharmonicity for physical modeling of dispersive strings," IEEE Trans. Speech Audio Process. 7, 597-601. 